\title{
Addressing the Conflict of Interest between Aggregators and DSOs in Deregulated Energy Markets
}

Heinrich, Carsten; Ziras, Charalampos; You, Shi; Bindner, Henrik W.

Published in:

Proceedings of the 52nd International Universities' Power Engineering Conference

Link to article, DOI:

10.1109/UPEC.2017.8231901

Publication date:

2017

Document Version

Peer reviewed version

Link back to DTU Orbit

Citation (APA):

Heinrich, C., Ziras, C., You, S., \& Bindner, H. W. (2017). Addressing the Conflict of Interest between Aggregators and DSOs in Deregulated Energy Markets. In Proceedings of the 52nd International Universities' Power Engineering Conference IEEE. https://doi.org/10.1109/UPEC.2017.8231901

\section{General rights}

Copyright and moral rights for the publications made accessible in the public portal are retained by the authors and/or other copyright owners and it is a condition of accessing publications that users recognise and abide by the legal requirements associated with these rights.

- Users may download and print one copy of any publication from the public portal for the purpose of private study or research.

- You may not further distribute the material or use it for any profit-making activity or commercial gain

- You may freely distribute the URL identifying the publication in the public portal 


\title{
Addressing the Conflict of Interest between Aggregators and DSOs in Deregulated Energy Markets
}

\author{
Carsten Heinrich, Charalampos Ziras, Shi You, Henrik W. Bindner \\ Department of Electrical Engineering \\ Technical University of Denmark \\ \{cahei, chazi, sy, hwbi\}@elektro.dtu.dk
}

\begin{abstract}
This paper investigates potential conflicts of interest between distribution system operators (DSOs) and aggregators. We propose a method to quantify the allowed operating range of residential flexible loads in a local distribution network. The calculated bounds can be used to formulate DSO services, tradable on a potential DSO service market platform. Aggregators are considered, concentrating thermostatically controlled loads and electric vehicles with vehicle2grid technology in order to perform arbitrage on the power market and to offer ancillary services.

Index Terms-Power distribution, Demand-side management, Load flow
\end{abstract}

\section{NOMEnClature}

\section{Parameter}

$\Delta t \quad$ Modelling timestep

$\eta^{+} \quad$ EV charging efficiency

$\eta^{-} \quad$ EV discharging efficiency

$\pi \quad$ Spot price

$C \quad$ Household internal heat capacity

COP Coefficient of Performance

$E^{\mathrm{kWh}} \quad$ Battery energy storage capacity

$R \quad$ Thermal resistance of household walls

$T^{\text {out }} \quad$ Indoor temperature

Sets

$\Omega \quad$ Set of scenarios, indexed as $\omega$

$E V \quad$ Set of electric vehicles, indexed as $e$

$F \quad$ Set of flexible loads, indexed as $f$

$H P \quad$ Set of heat pumps, indexed as $h$

$T \quad$ Set of timesteps, indexed as $t$

\section{Variables}

E Battery energy content

$I^{\text {rat }} \quad$ Current rating of power equipment

$I_{i j} \quad$ Current flowing from node $i$ to node $j$

$P^{+} \quad$ Electric vehicle charging power

$P^{-} \quad$ Electric vehicle discharging power

$P^{\text {tot }} \quad$ Total power consumption of flexible loads

$P_{\max }^{\text {tot }} \quad$ Upper bound for total power consumption

$P_{\min }^{\text {tot }} \quad$ Lower bound for total power consumption

$P_{f l} \quad$ Power consumption of flexible load

$P_{h} \quad$ Heat pump power consumption

$T^{\text {in }} \quad$ Indoor temperature

$V^{\text {nom }} \quad$ Nominal voltage magnitude

978-1-5386-2344-2/17/\$31.00 (C)2017 IEEE

\section{INTRODUCTION}

The improvements in information and communication technology, the deployment of smart meters and the electrification of the transportation and the heating sectors have led to the development of a new entity in the electricity system. Aggregators, who contract and control populations of electric loads can minimize overall costs according to day ahead market prices or may contribute to the overall network stability by offering ancillary services to the transmission system operator (TSO). While this business has so far mainly focused on large industrial customers, aggregators with portfolios consisting of residential loads have also appeared on the market. A notable case is the Swiss aggregator Tiko, which controls residential water boilers, heat pumps and resistive heaters of over ten thousand customers [1].

While this practice certainly grants benefits for the electricity grid as a whole, the coordinated and simultaneous control of residential consumption might at times conflict with the interests of local distribution system operators (DSOs). Under normal conditions, the stochasticity of individual household power consumption smooths out the aggregated consumption of all customers connected to a feeder. As the aggregator business model grows, coordinated control (e.g. due to day ahead cost minimization) could significantly increase the occurring power flows through the synchronization of power consumption. Thus, load forecasting becomes much more challenging and DSOs may face additional problems in keeping voltages in the allowed ranges and in avoiding overloading of network equipment.

The area of demand side participation has gotten a lot of attention in recent years. The possibility of flexible loads providing services to the grid has been widely discussed [2][5]. When evaluating the value of flexibility on the already existing markets, such as the balancing market or the spot market, limitations of flexible load and flexible generation through the distribution network are often neglected.

In Europe, system operators are not allowed to own generation units due to the principle of generation and distribution unbundling. System operators, that have access to information about the grid state are not able to control generation units or flexible loads directly. They have to 
interact with grid service providers through a market. While such a system is in place for TSO services and has proven to be beneficial, it is very challenging to also introduce it on the medium and low voltage level. DSO services should be designed to guarantee that voltage and thermal constraints are met and are therefore specific to certain points in the grid.

In Germany, a traffic light system has been proposed as a way of communication between aggregators and DSOs [6] in order to avoid aggregator induced overloading. The IDE4L project proposed two types of distribution services [7]. An option based service, which is traded day ahead and requires activation by the system operator, and a scheduled service, designed for forecasted congestion, for example during network maintenance work. The iPower project proposes five DSO services through active power control traded through a newly developed market clearing house [8]. A more detailed description of the proposed services can be found in [9].

This paper proposes a method to define a secure operating range of aggregators in a distribution feeder by solving a $\mathrm{AC}$ optimal power flow problem. The resulting bounds pose a limit on the total aggregated power consumption of all flexible loads in a considered area, which guarantees that grid constraints are not violated. Secure limits are found by considering a large number of load, weather and market scenarios. We estimate the value of the resulting power limitation services offered by a single aggregator to a DSO. The benefits of a freely controlled portfolio are compared with a scenario, where the aggregator actions are constrained, such that voltage and thermal limits in the distribution system are respected. The resulting cost difference can be interpreted as the aggregators provision cost of a power limitation service to the DSO.

Section II briefly discusses the main grid constraints of low voltage distribution networks. Section III introduces the used models for flexibility and proposes the methodology to define secure operating ranges for distribution system operators. Section IV] presents the case study, that was carried out in order to show the feasibility of the method. Finally, Section V] concludes the paper.

\section{Distribution Grid Planning And Operation}

In the past, low voltage networks used to have a passive role in power distribution and in the large majority of cases, power flowed from substations towards the individual customers. This allowed DSOs to design all distribution network components by simply considering worst case load scenarios and by assuming a constant power consumption increase during the operation years. But the situation in the low voltage grid has changed fundamentally. Instead of seeing a slight uniform and predictable power consumption increase of residential customers, their behaviour has become much more diverse, since residential customers have installed intermittent distributed generation, like photovoltaic units (PVs) and some customers have switched to more environmentally friendly solutions for transport and heating such as electric vehicles (EVs) and heat pumps. Households with PV units and EVs might at times feed power back into the grid. Hence, power flows in the distribution network have become more complex and are no longer unidirectional at all times. Aggregators, synchronizing residential loads may increase this effect. These new developments require to rethink the common grid planning and operational practices and make it necessary take customer flexibility into account.

There are two main constraints, that have to be fulfilled by DSOs during operation - the voltage constraint at customer nodes as well as the thermal constraint of individual grid equipment.

1) Voltage violations: According to the European norm 50160 [10], the voltage magnitude $V^{\mathrm{m}}$ at the connection point of residential customers has to lay in the range of $\pm 10 \%$ of the nominal voltage $V^{\text {nom }}$ of $230 V$

$$
0.9 V^{\text {nom }} \leq V^{\mathrm{m}} \leq 1.1 V^{\text {nom }}
$$

Since voltage drops in the direction of the power flow, it changes along a distribution feeder. Grids have historically been designed such, that voltages at the last transformer station lay slightly above nominal value and, given a constant unidirectional power flow, drop along the feeder to a value close to nominal voltage at all nodes. In a scenario with a high share of electric vehicles equipped with vehicle2grid technology, distributed generation like PV units and electric heating, the situation is much more complex and can not be solved by merely relying such old design rules.

2) Thermal violations: Electric power flows heat up the power distribution equipment, due to the inherent resistance of all materials. Since this power loss is a function of the flowing current $I$, all equipment has a current limit $I^{\text {rat }}$, that should not be exceeded, in order not to shorten the lifetime of the equipment

$$
-I^{\mathrm{rat}} \leq I \leq I^{\mathrm{rat}}
$$

The current is defined to be positive, when flowing in the direction of the load.

\section{Methodology}

\section{A. Feeder model}

In this work, a typical suburban low voltage distribution feeder with a transformer under high stress from [11] is considered. Figure 1 shows a sketch of the grid. A more detailed description of the grid can be found in [12]. The model represents a network in a village and has been developed, to represent a typical distribution feeder with a large load in southern Germany. We consider a future scenario, with a high share of electric vehicles and heat pumps. The feeder connects a total of 117 households and is modelled through a network of 236 nodes on nine branches. The parameters of the distribution grid are summarized in Table I. 


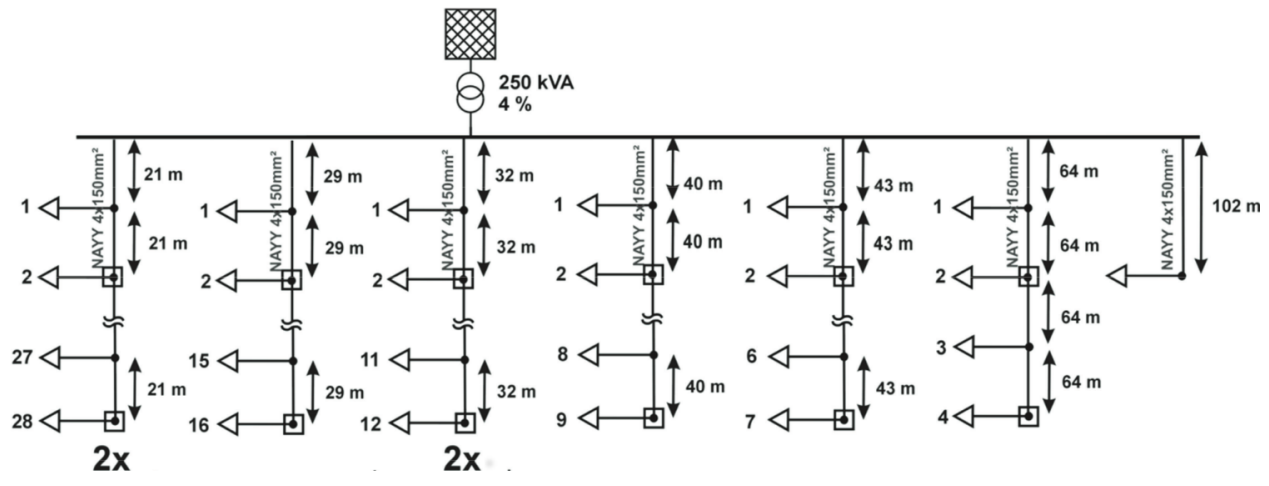

Fig. 1. Suburban low voltage distribution feeder with a transformer under high stress (Figure from [11])

\begin{tabular}{|c|c|c|}
\hline Parameter & Value & Unit \\
\hline Transformer rating & 250 & $k W$ \\
\hline Number of branches & 9 & \\
\hline Main line type & NAYY $4 \times 150 \mathrm{~mm}^{2}$ & \\
\hline NAYY $4 \times 150 \mathrm{~mm}^{2}$ resistance & 0.164 & $\Omega / k m$ \\
\hline NAYY $4 \times 150 \mathrm{~mm}^{2}$ impedance & 0.073 & $\Omega / k m$ \\
\hline NAYY $4 \times 150 \mathrm{~mm}^{2}$ capacitance & 0.5 & $\mu F / k m$ \\
\hline NAYY 4x150 $\mathrm{mm}^{2}$ rating & $322 \sqrt{3}$ & A \\
\hline Line to household & NAYY $4 \times 50 \mathrm{~mm}^{2}$ & \\
\hline NAYY $4 \times 50 \mathrm{~mm}^{2}$ resistance & 0.641 & $\Omega / k m$ \\
\hline NAYY 4x50 $\mathrm{mm}^{2}$ impedance & 0.078 & $\Omega / k m$ \\
\hline NAYY $4 \times 50 \mathrm{~mm}^{2}$ capacitance & 0.32 & $\mu F / k m$ \\
\hline NAYY $4 \times 50 \mathrm{~mm}^{2}$ rating & $275 \sqrt{3}$ & A \\
\hline cable connecting households & NAYY $4 \times 50 \mathrm{~mm}^{2}$ & \\
\hline Households on branch 1 and 2 & 28 & \\
\hline Cable length to PCC & 21 & $m$ \\
\hline Distance between PCC of households & $15 \& 31$ (alternating) & $m$ \\
\hline Households on branch 3 & 16 & \\
\hline Cable length to PCC & 29 (alternating) & $m$ \\
\hline Distance between PCC of households & $15 \& 31$ (alternating) & $m$ \\
\hline Households on branch 4 and 5 & 12 & \\
\hline Cable length to PCC & 32 & $m$ \\
\hline Distance between PCC of households & $15 \& 31$ (alternating) & $m$ \\
\hline Households on branch 6 & 9 & \\
\hline Cable length to PCC & 40 & $m$ \\
\hline Distance between PCC of households & $15 \& 31$ (alternating) & $m$ \\
\hline Households on branch 7 & 7 & \\
\hline Cable length to PCC & 43 & $m$ \\
\hline Distance between PCC of households & $15 \& 31$ (alternating) & $m$ \\
\hline Households on branch 8 & 4 & \\
\hline Cable length to PCC & 64 & $m$ \\
\hline Distance between PCC of households & $15 \& 31$ (alternating) & $m$ \\
\hline Households on branch 9 & 1 & \\
\hline Cable length to PCC & 102 & $m$ \\
\hline Distance between PCC of households & 15 & $m$ \\
\hline
\end{tabular}

TABLE I

SUMMARY OF DISTRIBUTION GRID PARAMETERS.

\section{B. Household model}

Households are modelled as power draws from nodes. Each household consists of a variable load profile, defined by connected heat pumps and electric vehicles, as well as an inflexible load part. The inflexible load part is modelled with the load generator from [13].

1) Heat pump model: The dynamics of the indoor temperature are modelled with a RC-model. The indoor temperature $T_{t, h}^{\text {in }}$ at time step $t$ and heat pump $h$ behaves according to

$$
\begin{aligned}
T_{t, h}^{\text {in }}=T_{t-1, h}^{\text {in }}+\Delta t \frac{P_{t, h} C O P_{h}}{C_{h}} & \\
& -\left(T_{t-1, h}^{\text {in }}-T_{t-1}^{\text {out }}\right) \frac{R_{h}}{C_{h}} \Delta t .
\end{aligned}
$$

Here $\Delta t$ represents the considered time step, $P_{t, h}$ is the heat pump power, $C O P_{h}$ stands for the coefficient of performance, $C_{h}$ is the household heat capacity, $T^{\text {out }}$ is the ambient temperature and $R_{h}$ is the effective thermal resistance. The indoor temperature is constrained by

$$
T^{\min } \leq T_{t, h}^{\mathrm{in}} \leq T^{\max }
$$

defining the comfort zone of the inhabitants. Instead of initializing the indoor temperature, the indoor temperature of the first time step is set to be equal to the last indoor temperature of the simulation horizon. This procedure simulates, that the indoor temperature was already controlled before the first time step. The power consumption of the heat pumps $P_{t, h}$ is constrained by the heat pump power rating $P_{h}^{\max }$ :

$$
0 \leq P_{t, h} \leq P_{h}^{\max } .
$$

Data for the individual household sizes has been provided by the project EcoGrid2.0 in the form of heated area in $\mathrm{m}^{2}$. Typical household heat capacities $C_{h}$ have been calculated by using values for heat capacity per square meter from [14]. Similar the effective wall heat resistance has been created with values from [15]. In order to calculate floor, roof, window and wall area, it was assumed, that the heated area has the shape of a square, the ceiling is $2.5 \mathrm{~m}$ high, and window area is $16 \%$ of the overall wall area.

2) Electric vehicle model: Electric vehicles are modelled as batteries, connected to the household nodes and equipped with vehicle2grid technology. Since the focus of a DSO is not to model the individual households accurately, but rather to get an overall idea of the grid state, a fairly simple EV-model is used here.

$$
E_{t, e}=E_{t-1, e}+\Delta t\left(P_{t, e}^{+} \eta^{+}+P_{t, e}^{-} \frac{1}{\eta^{-}}\right),
$$

where 


\begin{tabular}{cc|cc}
\hline \multicolumn{4}{c}{ Simulation Parameters } \\
Parameter & Value & Parameter & Value \\
\hline$t_{\text {arrive }}(h)$ & $\mathbb{U}[7,11]$ & $C O P(-)$ & $\mathbb{U}[3,4]$ \\
$t_{\text {leave }}(h)$ & $\mathbb{U}[16,21]$ & $E^{\mathrm{kWh}}(k W h)$ & 20 \\
$S O C_{\text {arrive }}(-)$ & $\mathbb{U}[0.1,0.6]$ & $T^{\min }\left({ }^{\circ} C\right)$ & 21.5 \\
$\eta^{+}(-)$ & $\mathbb{U}[0.85,0.9]$ & $T^{\max }\left({ }^{\circ} C\right)$ & 22.5 \\
$\eta^{-}(-)$ & $\mathbb{U}[0.85,0.9]$ & $\Delta t(s)$ & 900 \\
\hline
\end{tabular}

TABLE II

SUMMARY OF ELECTRIC VEHICLE AND HEAT PUMP MODEL PARAMETERS

$$
\begin{aligned}
& P_{e}^{\min } \leq P_{t, e}^{-} \leq 0 \\
& 0 \leq P_{t, e}^{+} \leq P_{e}^{\max }
\end{aligned}
$$

Here $E$ stands for the energy content of the vehicle battery. $P^{+}$ and $P^{-}$represent charging and discharging power respectively, which are limited by the battery power rating $P_{e}^{\min }$ and $P_{e}^{\max }$. $\eta^{+}$and $\eta^{-}$are the charging and discharging efficiency. Since a battery can not charge and discharge at the same time, an additional constraint is enforced,

$$
P_{t, e}^{+} P_{t, e}^{-}=0,
$$

such that one of the two variables is always zero. The state of charge $S O C$ is defined as the ratio of energy content $E$ and energy storage capacity $E^{\mathrm{kWh}}$

$$
S O C_{t, e}=\frac{E_{t, e}}{E_{e}^{\mathrm{kWh}}},
$$

where

$$
0 \leq S O C_{t, e} \leq 1 .
$$

Similar to the indoor temperature, the SOC is not initialized, but the last value of the modelled time period is set to be equal to the initial state. $P_{e}^{\max }=-P_{e}^{\min }$ is assumed to be $3.68 \mathrm{~kW}$, corresponding to a 16 Ampere fuse. Further we assume, that all vehicles are used once a day. The departure time $t_{\text {leave }}$ and the arrival time $t_{\text {arrive }}$ are chosen randomly. The SOC for departure hour is set to be greater than $60 \%$, hence,

$$
S O C_{t_{\text {leave }}, e} \geq 0.6 \text {. }
$$

Upon return, the SOC is chosen randomly. Parameters, that were used in the simulation, are summarized in Table II

\section{Calculation of operating range}

This Subsection describes the proposed method to ensure that the aggregator's control of a pool of flexible loads in a distribution grid does not lead to violations of the grid constraints. We define $P_{t}^{\text {tot }}$ to be the sum of the power consumption of all flexible loads in the grid at time $t$

$$
P_{t}^{\mathrm{tot}}:=\sum_{f \in F} P_{f, t},
$$

where $F$ is the set of flexible loads, indexed as $f$. The goal of the method is to define an upper limit for this aggregated power consumption. We formulate the following AC-optimal power flow problem, where we introduce an additional equation to fix one of the grid constraints to the edge of being violated. This is done for only one constraint at a time, then the problem is solved for every time step individually. For simplicity, we omit the index $t$.

$$
\min _{P_{f}}\left(\sum_{f} P_{f}\right)
$$

s.t.

$$
\begin{gathered}
P_{f}^{\mathrm{min}} \leq P_{f} \leq P_{f}^{\max } \forall f, \\
I_{i j}=I_{i j}^{\mathrm{rat}} \quad \text { or } \quad V_{i}^{\mathrm{m}}=0.9 V^{\mathrm{nom}}, \\
h(x)=0 .
\end{gathered}
$$

In this formulation, the individual load model is not taken into account. Flexible loads are simply represented by their power consumption constraint (15), such as Eq. (57, (7) or (8) and can take any value within these intervals. Eq. (16) sets one constraint as active. All other grid constraints stay the same. Eq. (17) represents all the standard $\mathrm{AC}$ power flow equations, active and reactive power balance at the nodes and power flow equations for every line and transformer as well as all the grid constraints.

The solution of the problem $P^{\text {tot* }}$ is the minimal total power consumption of the flexible load, such that the constraint, which was set to its edge, is violated. Therefore, this constraint can not be violated with a total power consumption smaller than $P^{\text {tot* }}$. If the problem is infeasible, the considered constraint can not be violated and can be omitted.

The problem is formulated and solved repeatedly, each time fixing a different grid constraint according to Eq. (16), hence each problem formulation leads to a different solution $P^{\text {tot }}$. The minimal value of all these individual solutions is defined as the new total power limit $P_{\max }^{\text {tot }}$.

$$
P^{\mathrm{tot}} \leq P_{\max }^{\mathrm{tot}}
$$

As long as Eq. (18) is fulfilled, no constraint can be violated. Since DSOs usually know the critical nodes and lines, the number of necessary calculatiions is limited.

The same procedure can be applied for upper voltage constraint and power flow towards the higher voltage levels in order to define a maximal power infeed by EVs or PVs.

$$
\max _{P_{f}}\left(\sum_{f} P_{f}\right) \text {, }
$$

s.t.

$$
\begin{gathered}
P_{f}^{\mathrm{min}} \leq P_{f} \leq P_{f}^{\mathrm{max}} \forall f, \\
I_{i j}=-I_{i j}^{\mathrm{rat}} \quad \text { or } \quad V_{i}^{\mathrm{m}}=1.1 V^{\mathrm{nom}}, \\
h(x)=0 .
\end{gathered}
$$

The areas, for which the individual power consumption limits are calculated can be chosen freely but have to be defined reasonably, in order to get helpful results. The smaller the areas are, the more optimal the possible allowed operational range will be. 


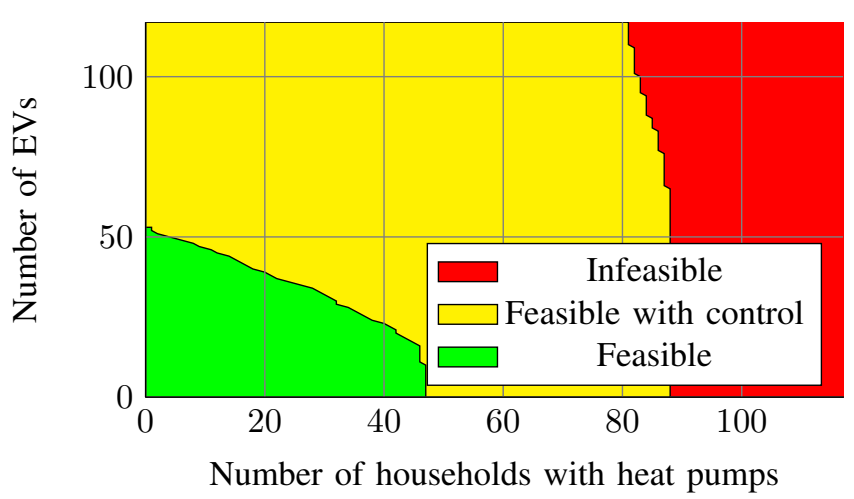

Fig. 2. Feasibility as function of penetration level of both EVs and heat pumps. The green area is always feasible, in the yellow area control actions are necessary. With a penetration level in the red area it is impossible to avoid violating grid constraints.

\section{CAse Study}

In this Section a simple case study is carried out in GAMS. We consider the grid introduced in Section III-A The critical constraint in this grid is the power rating of the $20 \mathrm{kV} / 400 \mathrm{~V}$ transformer. Figure 2 shows the feasible penetration levels depending on the control structure of the flexible devices. The green area represents the penetration levels of heat pumps and EVs where no violations occur in the grid and the aggregator can operate entirely unconstrained. In the yellow area, the aggregator violates the grid constraints, when it only optimizes its power consumption profile according to the spot market, but can operate the portfolio such, that no violations occur. The red area represents the region, where the aggregator is not able to respect both the customer comfort constraints as well as the grid constraints. Here, grid expansions are indispensable.

For this case study, we assumed, that 40 households in the grid are equipped with flexible heat pumps and 60 households own an electric vehicle. Those households are chosen randomly and are all controlled by a single aggregator, which has no information about the grid state, but perfect information about the loads. The simulations are carried out for 30 scenarios, consisting of 30 days in January. Spot price data from January 2015 of the Nord Pool market for Eastern Denmark has been used. The heat pump as well as outdoor temperature data originate from the Danish island Bornholm and were collected in the context of the EcoGrid EU project during the same time period. We define four sets:

- $T$ is the set of time steps, indexed as $t$,

- $\Omega$ is the set of scenarios, indexed as $\omega$,

- $E V$ is the set of electric vehicles, indexed as $e$,

- $H P$ is the set of heat pumps, indexed as $h$.

Three different optimization steps are carried out. First, a non-linear program is solved, which minimizes the energy acquisition cost of an aggregator's portfolio and respects the individual constraints of the flexible loads to guarantee customer comfort. The simulation is carried out for 24 hours with a 15 minute time step. During this step, the grid is

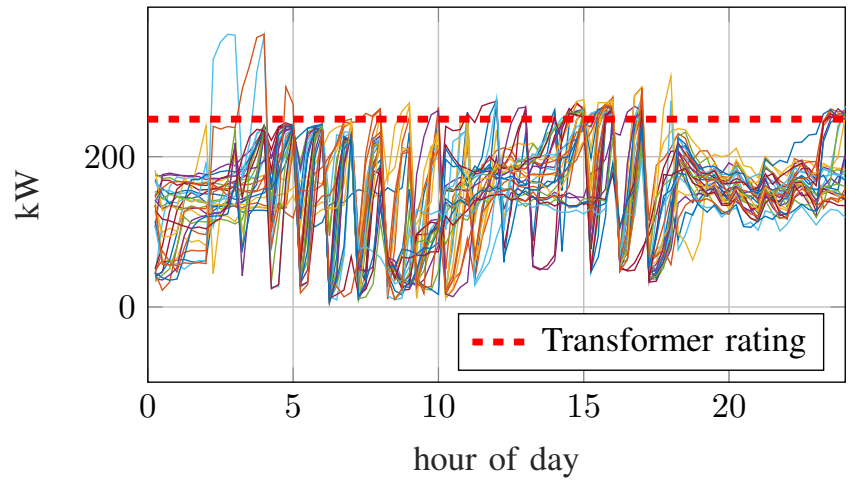

Fig. 3. Line flow through voltage transformer for the 30 scenarios. The aggregator does take grid constraints into account. The transformer constraint is violated regularly with power flows up to $360 \mathrm{~kW}$.

neglected, in order to model the unconstrained behaviour of an aggregator.

$$
\begin{gathered}
\min _{P_{\omega, t, e}^{+}, P_{\omega, t, e}^{-}, P_{\omega, t, h}} \sum_{\omega \in \Omega} \sum_{t \in T} P_{t, \omega}^{\mathrm{tot}} \pi_{t, \omega} \\
P_{t, \omega}^{\mathrm{tot}}=\sum_{e \in E V}\left(P_{\omega, t, e}^{+}+P_{\omega, t, e}^{-}\right)+\sum_{h \in H P} P_{\omega, t, h}
\end{gathered}
$$

s.t.

$$
\begin{aligned}
& \text { Eq. (3) to (5), } \forall h \in H P \\
& \text { Eq. (6) to (12), } \forall e \in E V
\end{aligned}
$$

The results of the power flowing through the transformer are shown in Fig. 3 for all 30 scenarios. The power flow exceeds the transformer rating regularly and in a non negligible manner, reaching values of up to $360 \mathrm{~kW}$.

In the second step, the aggregator's operating range is calculated according to the procedure presented in Section III-C This is done for each 15 minute time step of the 30 considered scenarios individually. The resulting minimal total power consumption of the flexible load in the grid is shown in Fig. 4 for all 30 scenarios. We define the minimum of all results as the new maximal power $P_{\max }^{\text {tot }}$.

$$
P_{\max , t}^{\mathrm{tot}}:=\min _{\omega}\left(P_{\omega, t}^{\mathrm{tot} *}\right) \forall t \in T
$$

This result is depicted as the blue line in Fig. 4 . In the final step, we run the aggregator optimization with the additional constraint, enforcing that the total power consumption of all heat pumps and EVs lays below the newly defined curve, which defines a maximal power consumption for every timestep throughout the day.

$$
\begin{gathered}
\min _{P_{\omega, t, e}^{+}, P_{\omega, t, e}^{-}, P_{\omega, t, h}} \sum_{\omega \in \Omega} \sum_{t \in T} P_{t, \omega}^{\mathrm{tot}} \pi_{t, \omega} \\
P_{t, \omega}^{\mathrm{tot}}=\sum_{e \in E V}\left(P_{\omega, t, e}^{+}+P_{\omega, t, e}^{-}\right)+\sum_{h \in H P} P_{\omega, t, h}
\end{gathered}
$$

s.t.

$$
P_{t, \omega}^{\mathrm{tot}} \leq P_{\max , t}^{\mathrm{tot}} \forall \omega \in \Omega, t \in T
$$




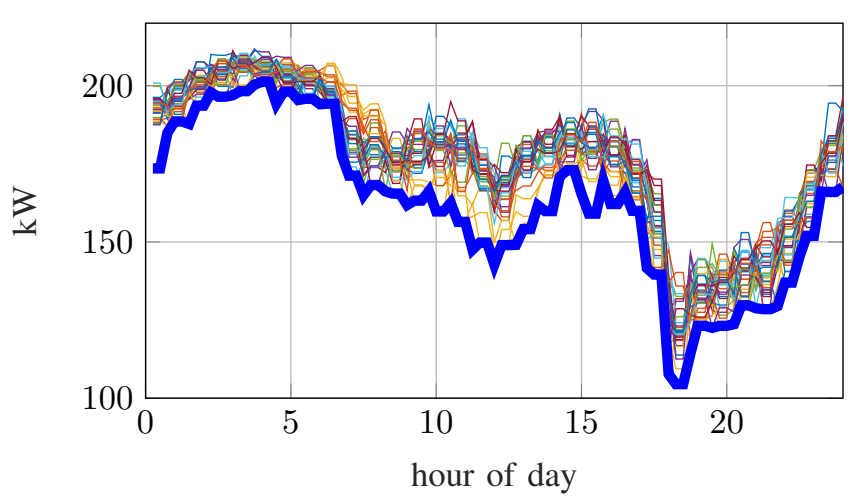

Fig. 4. Maximal total power consumption of the pool of flexible loads as a function of time during each scenario. The minimal value of all 30 results is chosen as the new maximal total power consumption.

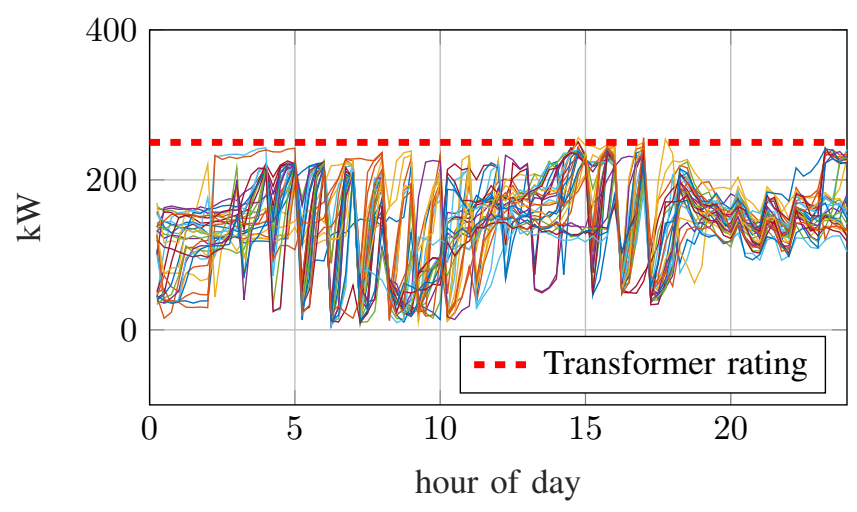

Fig. 5. Transformer power flow results for the 30 considered scenarios with the total flexible load power constraint.

Eq. (3) to (5), $\forall h \in H P$

Eq. (6) to 12, $\forall e \in E V$

The result of this optimization step can be seen in Fig. 5 , As the graph shows, the transformer rating is now respected, while at the same time, the aggregator is still able to optimize its portfolio according to the prices. The simulation step was carried out with only one additional constraint for the total portfolio power consumption. Table III shows the total power acquisition costs for the aggregator during the simulated month. Only a very small cost increase could be observed, which can be explained by the fact, that the aggregators overall allowed operating range has only been decreased insignificantly. During most time steps, the aggregator is not affected by the introduced limits.

\begin{tabular}{cc}
\hline Scenario & Aggregator Costs \\
Unconstrained Aggregator & $1376.3 €$ \\
Aggregator with total power limit & $1377.3 €$ \\
\hline
\end{tabular}

TABLE III

SUMMARY OF COSTS FOR THE TWO DIFFERENT SCENARIOS.

\section{CONCLUSION}

This work presented a method to define the secure operating range of aggregators in low voltage networks. The feasibility of the procedure has been shown in a simple case study. The resulting operating ranges ensure that grid constraints are not violated and can be used to define DSO services, which a DSO requests from aggregators or can serve as a capacity, which aggregators have to acquire, in order to be active in a distribution grid. The operating ranges can be calculated by DSOs offline using historical data for loads and weather and can be adjusted to individual areas.

\section{ACKNOWLEDGEMENT}

The authors would like to acknowledge the financial support of the EUDP project, EcoGrid 2.0, No 64015-002.

\section{REFERENCES}

[1] Swisscom Energy Solutions AG. tiko - official web representation, 2017 (accessed June 20th, 2017). 1

[2] He Hao, Borhan M Sanandaji, Kameshwar Poolla, and Tyrone L Vincent. Aggregate flexibility of thermostatically controlled loads. IEEE Transactions on Power Systems, 30(1):189-198, 2015. 1

[3] George Wenzel, Matias Negrete-Pincetic, Daniel E Olivares, Jason MacDonald, and Duncan S Callaway. Real-time charging strategies for an electric vehicle aggregator to provide ancillary services. IEEE Transactions on Smart Grid, 2017.

[4] Theodor Borsche and Göran Andersson. A review of demand response business cases. In Innovative Smart Grid Technologies Conference Europe (ISGT-Europe), 2014 IEEE PES, pages 1-6. IEEE, 2014.

[5] Duncan S Callaway and Ian A Hiskens. Achieving controllability of electric loads. Proceedings of the IEEE, 99(1):184-199, 2011. 1

[6] Henriette Zoeller, Markus Reischboeck, and Sylwia Henselmeyer. Managing volatility in distribution networks with active network management. 2016. 2

[7] Sami Repo, Ferdinanda Ponci, Davide Della Giustina, Amelia Alvarez, Cristina Corchero Garcia, Zaid Al-Jassim, Hortensia Amaris, and Anna Kulmala. The ide4l project: Defining, designing, and demonstrating the ideal grid for all. IEEE Power and Energy Magazine, 15(3):41-51, 2017. 2

[8] Chunyu Zhang, Yi Ding, Jacob Ostergaard, Henrik W Bindner, Niels Christian Nordentoft, Lars Henrik Hansen, Poul Brath, and Peder Dybdal Cajar. A flex-market design for flexibility services through ders. In Innovative Smart Grid Technologies Europe (ISGT EUROPE), 2013 4th IEEE/PES, pages 1-5. IEEE, 2013. 2

[9] NC Nordentoft, Y Ding, LH Hansen, P Dybdal Cajar, P Brath, HW Bindner, and $\mathrm{C}$ Zhang. Development of a dso-market on flexibility services. iPower, http://ipower-net. dk/Publications. aspx, Tech. Rep, 2013. 2

[10] EN Standard. 50160. Voltage Characteristics of electricity supplied by public distribution networks, Cenelec, 2010. 2

[11] Georg Kerber and Rolf Witzmann. Statistische Analyse von NSVerteilungsnetzen und Modellierung von Referenznetzen-Netzklassen mit quantitativen Unterscheidungsmerkmalen. Elektrizitatswirtschaft, 107(6):22, 2008. 23

[12] Georg Kerber. Aufnahmefhigkeit von Niederspannungsverteilnetzen $\mathrm{fr}$ die Einspeisung aus Photovoltaikkleinanlagen. $\mathrm{PhD}$ thesis, Mnchen, Technische Universitt Mnchen, Diss., 2011, 2011. 2

[13] Christof Bucher. Analysis and Simulation of Distribution Grids with Photovoltaics. PhD thesis, ETH Zurich, 2014. 3

[14] Comité Europeo de Normalización. EN ISO 13790: Energy Performance of Buildings: Calculation of Energy Use for Space Heating and Cooling (ISO 13790: 2008). CEN, 2008. 3

[15] Paolo Zangheri, Roberto Armani, Marco Pietrobon, Lorenzo Pagliano, M Fernandez Boneta, and A Müller. Heating and cooling energy demand and loads for building types in different countries of the eu. Polytechnic University of Turin, end-use Efficiency Research Group, 2014. 3 